\title{
Integrando as Plataformas FIWARE e KNoT para o Desenvolvimento de Aplicações de Internet das Coisas
}

\author{
César Perdigão Batista, Pedro Victor Silva, Thais Batista, Everton Cavalcante
}

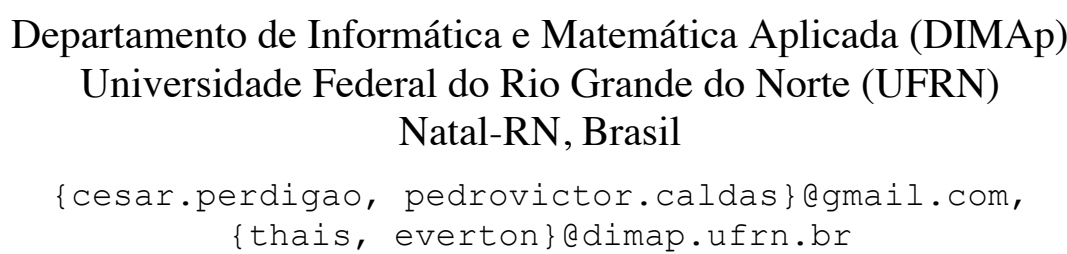

\begin{abstract}
Middleware platforms have been used as underlying infrastructure to ease development of Internet of Things (IoT) applications. FIWARE is a platform that has stood out in this scenario as an open, extensible solution for application development, but it supports only devices with native Internet connection. On the other hand, the KNoT platform allows virtualizing physical devices that do not have IP communication with minimal development effort. This paper presents the integration of the two platforms through the development of a component compatible with the FIWARE standard interface, inserted into KNoT aiming at enhancing their use for IoT applications.
\end{abstract}

Resumo. Plataformas de middleware têm sido utilizadas como infraestrutura subjacente para facilitar o desenvolvimento de aplicações de Internet das Coisas (IoT). A FIWARE é uma plataforma que tem se destacado nesse cenário como uma solução aberta e extensível para desenvolvimento de aplicações, porém ela admite apenas dispositivos com conexão nativa à Internet. Por outro lado, a plataforma KNoT possibilita a virtualização de dispositivos físicos que não possuem comunicação via IP com mínimo esforço de desenvolvimento. Este artigo apresenta a integração dessas duas plataformas por meio do desenvolvimento de um componente compatível com a interface padrão da FIWARE, inserido na KNoT para potencializar o uso de ambas as plataformas para aplicações de IoT.

\section{Introdução}

A multiplicação de dispositivos computacionais de diversas proporções possibilita a criação de um espaço virtual massivo que, quando integrado de maneira eficiente a objetos do mundo físico, permite a exploração de uma variedade de aplicações envolvendo usuários e dispositivos. Dessa forma, é interessante que tanto hardware quanto software aglutinem-se para que dispositivos conectados ofereçam serviços e atuem de forma incisiva nas duas realidades. O paradigma de Internet das Coisas (do Inglês Internet of Things - IoT) [Atzori et al. 2010, Miorandi et al. 2012] abrange desde como a infraestrutura de hardware se organiza até a abstração em diferentes camadas de software para conectar objetos do mundo real (coisas) às redes de computadores.

Aplicações de IoT demandam plataformas que visem facilitar o seu processo de desenvolvimento, o qual pode envolver a integração de uma diversidade de dispositivos heterogêneos, com capacidades variadas, meios de transmissão de dados e protocolos de comunicação distintos. A literatura apresenta várias plataformas de middleware que servem como infraestrutura subjacente ao desenvolvimento de aplicações de IoT 
[Mineraud et al. 2016, Ngu et al. 2017]. Nesse contexto, a Comissão Europeia disponibilizou a plataforma FIWARE ${ }^{1}$, um middleware open-source desenvolvido para contribuir com a criação de tecnologias voltadas para a Internet do Futuro e que é capaz de atender a uma ampla gama de requisitos considerados relevantes para plataformas de IoT [Batista et al. 2016]. A FIWARE provê diversos componentes extensíveis e reutilizáveis que podem facilitar o desenvolvimento de aplicações, os chamados habilitadores genéricos (do Inglês generic enablers - GEs). Tais componentes seguem o modelo de especificação NGSI (Next Generation Service Interfaces) ${ }^{2}$ para padronizar a troca de informações e permitir a interoperabilidade entre eles.

Também visando oferecer facilidades para interoperabilidade e gerenciamento de dispositivos para o desenvolvimento de aplicações de IoT, o Centro de Estudos e Sistemas Avançados do Recife (CESAR) concebeu a plataforma KNoT ${ }^{3}$. Baseada em hardware de baixo custo e software com implantação simplificada, a KNoT propõe-se a permitir interoperabilidade entre plataformas e dados de diversos dispositivos, oferecendo protocolos de diferentes camadas do modelo OSI com o intuito de possibilitar adaptabilidade às necessidades de cada aplicação em particular. Essa plataforma promove ainda a virtualização de dispositivos físicos para que estes possam ser acessados por outras plataformas, usuários e aplicações.

Ainda que seja possível conectar dispositivos de IoT à FIWARE, existem dificuldades para lidar com a inerente heterogeneidade a nível de hardware, uma vez que a própria plataforma é focada em prover módulos de software para facilitar o desenvolvimento de aplicações. Os GEs de IoT da FIWARE não oferecem suporte satisfatório para dispositivos que não possuem em seu hardware capacidade de conexão direta à Internet. Portanto, no ecossistema da FIWARE, a plataforma KNoT poderia ser inserida com o papel de prover novos meios de comunicação entre os dispositivos, a fim de facilitar a integração de variados objetos físicos em um ambiente virtual e interoperável. A KNoT destaca-se pela facilidade de implantação de novos dispositivos ao ambiente, exigindo mínima intervenção por parte do desenvolvedor, o qual basicamente terá de implementar as funções de leitura e escrita dos dispositivos a nível de microcontrolador e registrá-los no seu gateway. Para isso, microcontroladores comuns do mercado, compatíveis com os mais diversos dispositivos, comunicam-se através de radiofrequência com o gateway da KNoT que, por sua vez, fornece os dados providos por tais dispositivos para plataformas, aplicações e/ou usuários que deles irão fazer uso.

No intuito de suplantar as limitações mencionadas, este trabalho apresenta a integração da plataforma KNoT com o ecossistema FIWARE para que dispositivos a serem utilizados por uma aplicação construída sobre a FIWARE possam fazer uso das facilidades de implantação e acesso a dispositivos físicos providas pela KNoT. Do lado da KNoT, essa integração faz com que seja possível a utilizar os diversos mecanismos providos pela FIWARE para o desenvolvimento de aplicações de IoT. Além de apresentar a integração realizada no contexto deste trabalho, o artigo apresenta uma validação através do desenvolvimento de uma aplicação que explora o cenário integrado entre as duas plataformas.

\footnotetext{
${ }^{1}$ https://www.fiware.org/

${ }^{2}$ https://goo.gl/RqXk12

${ }^{3}$ https://www.knot.cesar.org.br/
} 
O restante deste artigo está organizado como segue. A Seção 2 apresenta brevemente as plataformas FIWARE e KNoT. A Seção 3 apresenta a solução desenvolvida neste trabalho para integração das duas plataformas. A Seção 4 ilustra o uso da integração FIWARE-KNoT no desenvolvimento de uma aplicação. Alguns trabalhos relacionados são discutidos na Seção 5. A Seção 6 traz algumas considerações finais e direções para trabalhos futuros.

\section{As Plataformas FIWARE e KNoT}

Apesar de tanto a FIWARE quanto a KNoT oferecem uma infraestrutura facilitadora para aplicações de IoT, o foco das duas plataformas não é o mesmo. Conectar objetos inteligentes a uma camada superior agregadora a partir da qual eles serão de fato acessados é apenas um dos módulos da FIWARE e isso já é um desafio por si só justamente pelo alto grau de heterogeneidade de tais objetos. A KNoT busca não apenas descomplicar essa barreira, mas também adicionar maior flexibilidade à configuração e instalação de dispositivos físicos pelo fato de dar suporte a outros meios de transmissão de dados entre eles (e.g., rádios subgigahertz, rádios Mesh, rádios de área ampla e baixa potência, Bluetooth, etc.). Nesta seção será apresentada uma visão geral dos elementos das plataformas FIWARE e KNoT e como eles podem ser utilizados para a integração de dispositivos físicos e aplicações de IoT.

\subsection{FIWARE}

A FIWARE é uma plataforma aberta, genérica e extensível voltada principalmente para dar suporte ao desenvolvimento de aplicações em cidades inteligentes. A FIWARE provê um conjunto de especificações disponíveis através de APIs abertas e está estruturada sobre componentes de software de alto nível, os GEs. Os GEs seguem o modelo de especificação NGSI para padronizar a troca de informações e permitir a interoperabilidade entre os componentes. No NGSI, as informações são estruturadas de forma genérica por meio de entidades de contexto que podem ser utilizadas para representar, de forma virtual, os elementos do mundo real, tais como um lugar, uma pessoa, um objeto, sensores, atuadores, etc. Um elemento de contexto possui um identificador, um tipo e uma lista de atributos, os quais possuem um nome, um tipo, um valor e uma lista de metadados, cada um deles com nome, tipo e valor. De forma prática, o contexto dos dispositivos é disponibilizado no Orion Context Broker (ou simplesmente Orion), o GE responsável por gerenciar o contexto das entidades. Uma vez que esses dados estejam no Orion, uma aplicação pode realizar a consulta de seu estado ou outros GEs podem ser por ela utilizados via NGSI.

A FIWARE provê alguns GEs que podem ser utilizados no desenvolvimento de aplicações de IoT. Dentre eles, o IoT Discovery e o IoT Broker oferecem um conjunto de funcionalidades e abstrações que facilitam o compartilhamento e o consumo de dados no âmbito da plataforma e não agem como fornecedores de dados dos dispositivos físicos ao Orion. Por sua vez, o Backend Device Management, apesar de ser um agente IoT que permite a conversão dos protocolos dos dispositivos e sensores (tais como MQTT, CoAP ou HTTP) para o padrão NGSI, limita-se a receber essas mensagens a nível de software através de rede Ethernet, convertê-las e encaminhá-las a um broker. Essas capacidades oferecidas pelos GEs de IoT do FIWARE são convenientes quando os equipamentos já estão instalados, porém não solucionam de maneira independente os problemas relacionados com a instalação física, meios de 
transmissão entre o dispositivo e o gateway, ou facilidades em prover conectividade para torná-los virtualizáveis.

\subsection{KNoT}

A Figura 1 ilustra a arquitetura da KNoT, mostrando como dispositivos físicos (referidos como devices ou KNoT Things), que não se conectam diretamente à Internet, são conectados através de um Gateway. Nessa arquitetura, os devices são compostos por um microcontrolador e um módulo de radiofrequência. $\mathrm{O}$ desenvolvedor pode conectar seus sensores e atuadores a este dispositivo principal para criar um device.

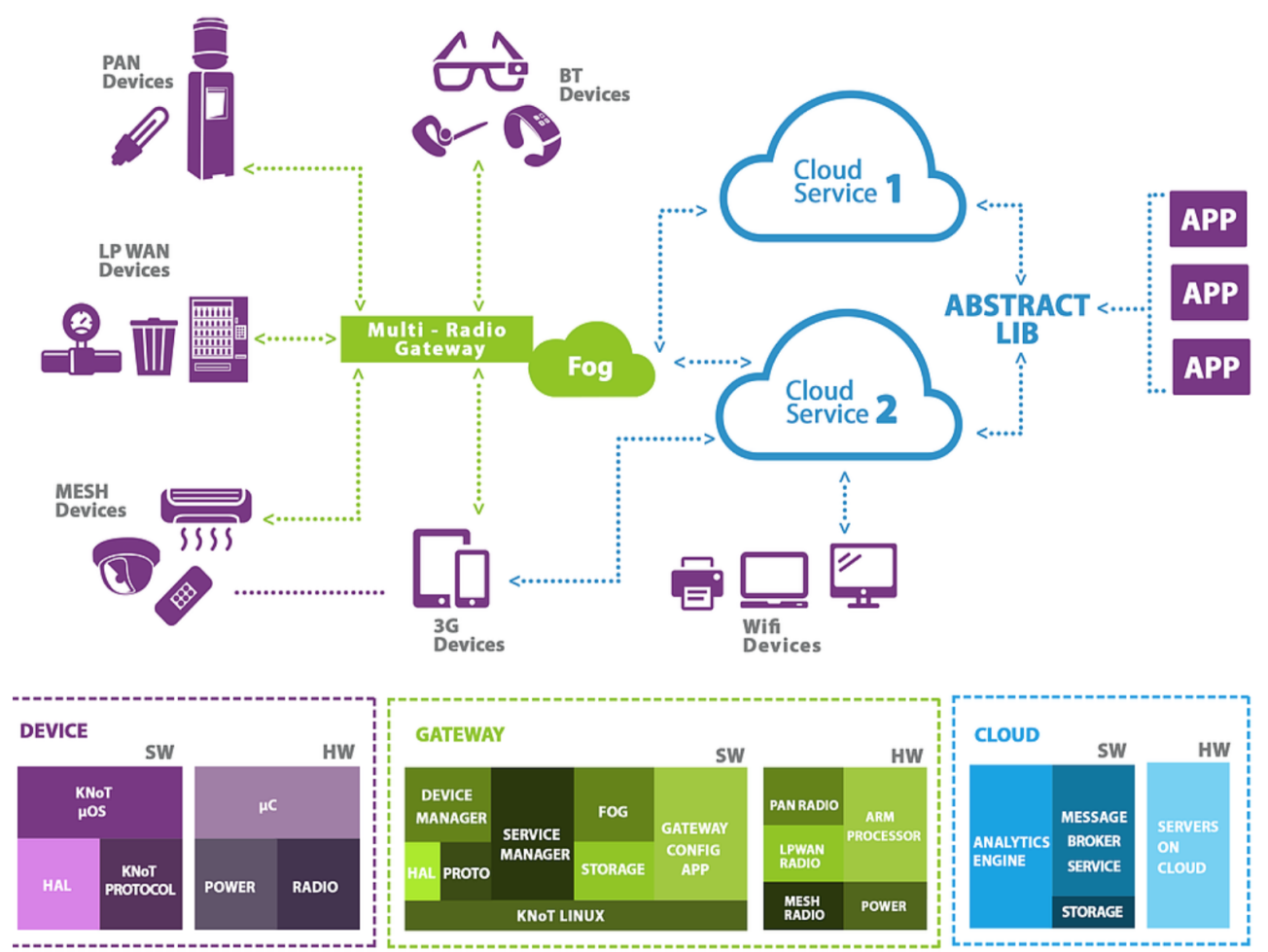

Figura 1. Arquitetura da plataforma KNoT versão 1.0 (https://www.knot.cesar.org.br/)

De maneira resumida, os macrocomponentes da plataforma são três, a saber, Things (devices), Gateway e Cloud. As Things, mais comumente constituídas por um dispositivo Arduino com sensores ou atuadores, conectam-se ao Gateway por meio de um transmissor de rádio para troca de dados. No caso do Gateway, elemento central que normalmente consiste em um dispositivo Raspberry Pi dotado de conexão Ethernet e transmissores de rádio nRF24L01 e/ou LoRa que traduz o protocolo das Things para JSON. O Gateway, que consiste em uma distribuição Linux embutida e personalizada chamada KNoT Linux, também oferece um módulo de software chamado WebUI, utilizado para gerenciar Things. Por fim, a Cloud é responsável por coletar os dados do Gateway e atua como um ponto de acesso remoto para as aplicações, além de ser responsável pelo roteamento de mensagens para aplicativos e dispositivos.

A prática de se usar um gateway como nó de comunicação entre os dispositivos e plataformas de nuvem é comum em IoT, pois são os gateways que normalmente orquestram os dispositivos agregados [Cavalcante et al. 2016]. Entretanto, o diferencial do KNoT é que os dispositivos instalados abaixo da camada do Gateway são abstraídos, não importando qual tipo de conexão física o sensor possui. Contanto que o dispositivo 
seja instalável em um microcontrolador compatível, os dados podem ser enviados para o Gateway e, portanto, serem virtualizados para camadas superiores. Isso é viabilizado pela comunicação entre a Thing e o Gateway ao permitir que novos meios de transmissão sem fio sejam empregados para fazer esse intermédio.

A flexibilidade referente a como os dados dos dispositivos podem ser transmitidos para o Gateway é interessante, já que estabelece uma perspectiva em que equipamentos que normalmente não tem conectividade à Internet possam ser integrados a ambientes de nuvem com facilidade e, com isso, diversas aplicações podem consultar seus dados e atuar sobre eles. Considerando que a ponte Thing-Gateway-Cloud é feita de maneira transparente, o desenvolvedor que utilizará a KNoT para construir uma aplicação apenas precisará se preocupar com a exposição dos dados dos dispositivos físicos, definindo o comportamento deles a nível de microcontrolador. Para isso, são oferecidas bibliotecas disponíveis para os mais variados tipos de hardware que determinam a operação e estabelecem a comunicação entre o microcontrolador e $o$ Gateway.

\section{Integração entre FIWARE e KNoT}

A plataforma KNoT propõe uma solução prática para a integração de dispositivos físicos heterogêneos sem comunicação via IP nativa. Dessa forma, a ideia de complementar esse atributo tornando-o mais flexível e exposto a um ambiente rico como o da FIWARE é proveitosa, visto que a KNoT passará a ter acesso ao ecossistema FIWARE, que oferece variadas ferramentas para o desenvolvimento de aplicações com diferentes funcionalidades. No entanto, para realizar essa integração, é necessário que as camadas inferiores da KNoT, responsáveis pela comunicação com os equipamentos físicos, sejam adaptadas para interagir com o Orion, componente central da FIWARE. A diferença de estrutura entre as duas plataformas exige a modificação de alguns componentes de menor granularidade da KNoT e a adição de um componente da FIWARE à KNoT para fazer com que as informações providas sejam compatíveis com o formato NGSI e endereçáveis ao Orion.

A Figura 2 ilustra a arquitetura da integração da KNoT com a FIWARE e seus componentes. Dentre esses componentes da arquitetura, a KNoT Thing Library, implementada na KNoT, possibilita que microcontroladores diversos estabeleçam comunicação com o Gateway. Os desenvolvedores precisam apenas usá-la configurando alguns dados e funções para que a comunicação seja feita. Feito isso, é necessário acessar o componente WebUI do Gateway, o qual atua no gerenciamento dos dispositivos vinculados ao Gateway, e se comunicar com o componente knotd para compartilhar informações sobre o registro de dispositivos e permitir a interação com eles. 


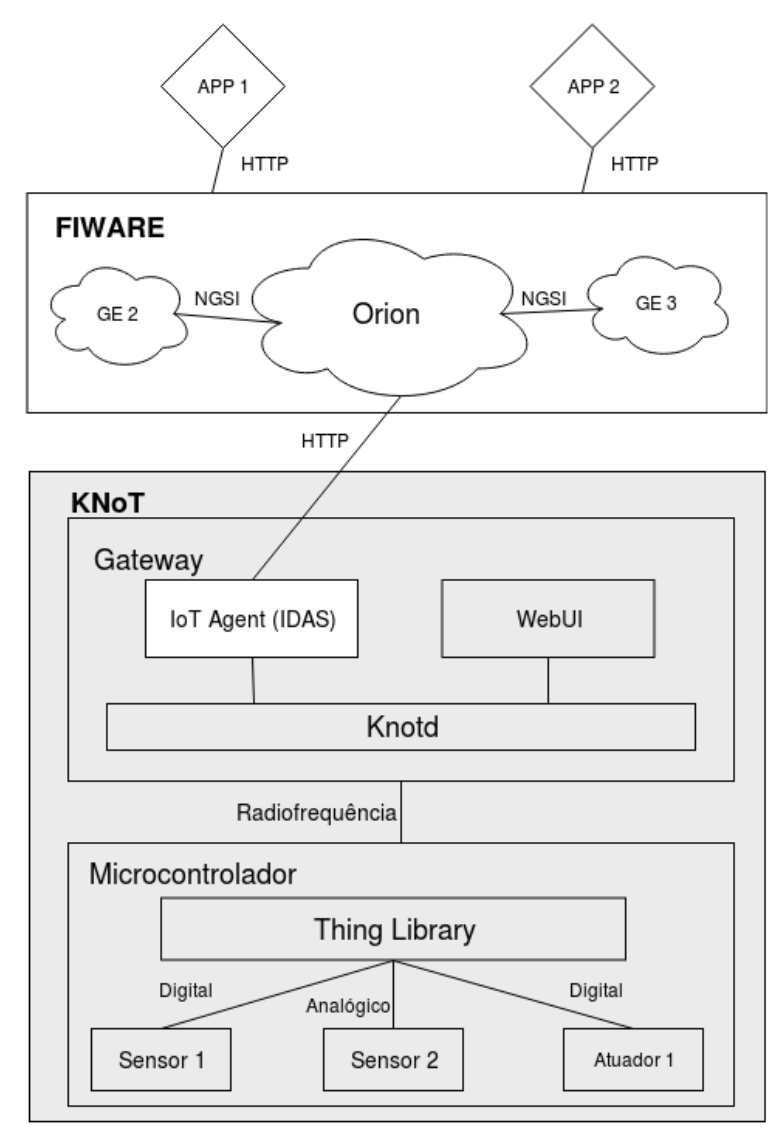

Figura 2. Arquitetura da integração entre KNoT e FIWARE

O knotd é o software executado no Gateway, que foi modificado para dar suporte à integração com a FIWARE e receber os dados dos equipamentos via rádio ou outras tecnologias de comunicação sem fio. Tais dados são traduzidos para o formato JSON antes de serem enviados ao IoT Agent, componente do Intelligence Data Advanced Solution (IDAS), uma implementação do GE Backend Device Management da FIWARE.

O IoT Agent foi desenvolvido e instanciado no Gateway para receber os dados do knotd e desempenhar toda a tarefa de cadastro, atualização, exclusão e leitura, exigidas para a produção de um contexto compatível com NGSI. Seu papel torna-se relevante pelo fato de ele não se limitar a criar um canal de comunicação com o Orion ou converter o protocolo adotado na KNoT para NGSI, mas também por oferecer serviços comuns ao ambiente da FIWARE, tais como lidar com aspectos de segurança da plataforma e enviar comandos. Além disso, ele assume os papeis de criar subscrições nas entidades do Orion para que o knotd seja avisado sempre que haja uma atualização e encaminha as ações ocorridas aos devidos dispositivos. Isso é possível devido à modificação do knotd, realizada neste trabalho, para que as mensagens provenientes dos dispositivos sejam traduzidas para uma estrutura compatível com a do Orion. Além disso, foi necessário elaborar um controle de mensagens entre o Orion e o knotd para tomar ações de acordo com as subscrições nas entidades do Orion. A Figura 3 ilustra a troca de mensagens do KNoT integrado com o Orion, exemplificando o cadastro de um dispositivo. 


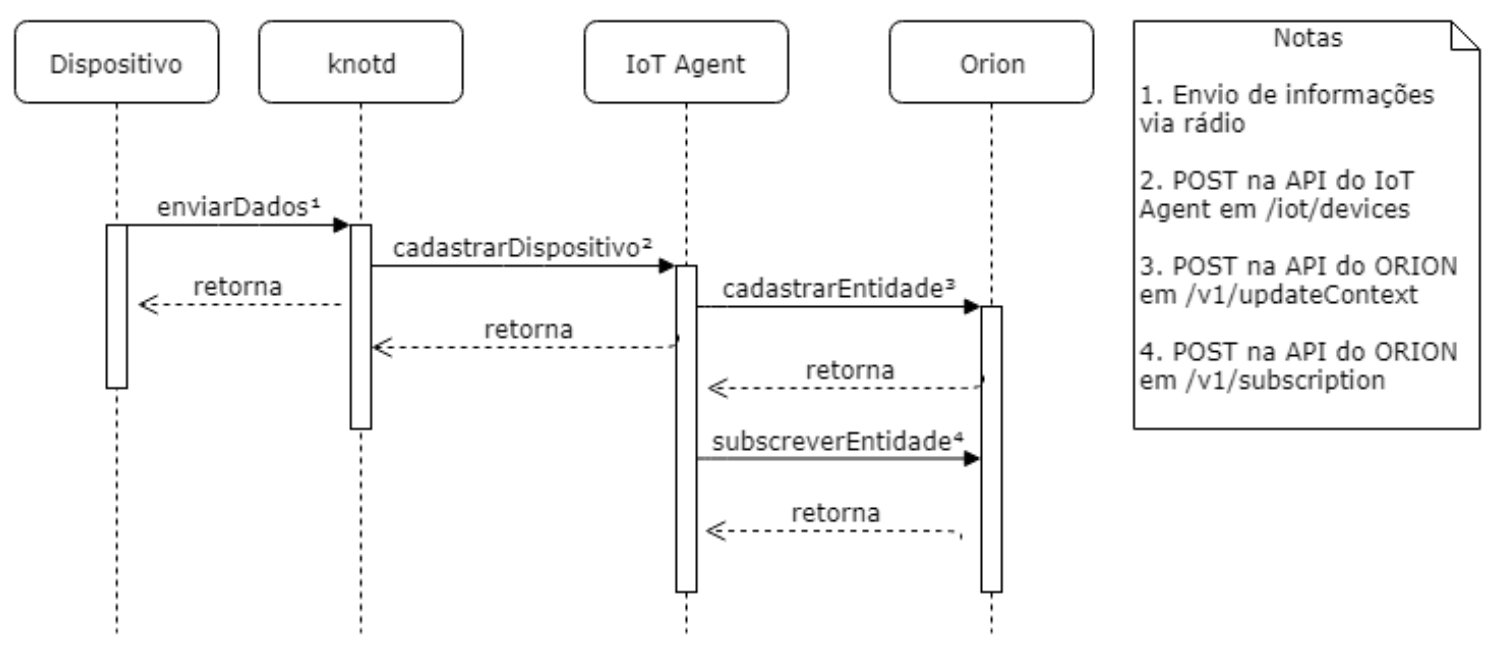

Figura 3. Diagrama de Sequência ilustrando a operação de cadastro de um dispositivo

Com a integração realizada, dispositivos que normalmente não poderiam ser inseridos no ecossistema FIWARE podem ser agora modelados como entidades no Orion e, consequentemente, ser acessados e utilizados por outros GEs e/ou por aplicações diversas construídas sobre a plataforma. O procedimento genérico que deve ser realizado por um desenvolvedor para que uma aplicação possa utilizar um dispositivo provido ao Orion pela KNoT consiste em: (i) conectar os sensores/atuadores do dispositivo e o transmissor de radiofrequência fisicamente ao microcontrolador; (ii) importar a biblioteca KNoT Thing Library no microcontrolador para definir o funcionamento do dispositivo e permitir que as entidades de contexto desejadas sejam criadas no Orion e o gerenciamento delas pela KNoT funcione; (iii) acessar o WebUI do Gateway para configurar o endereço do Orion a ser utilizado para registro de entidades; (iv) realizar a aceitação dos dispositivos disponíveis no WebUI, admitindo-os automaticamente no Orion, e; (v) usar a entidade cadastrada na aplicação que realiza operações HTTP no Orion.

\section{Validação}

De forma a validar a solução apresentada neste trabalho, foi desenvolvida a aplicação LightsOut, que controla a iluminação de uma sala (ver Figura 4). Nessa aplicação, uma lâmpada originalmente sem conexão à Internet está modelada como uma entidade de contexto no Orion da FIWARE e, como tal, contém informações sobre seu estado (ligada e desligada) e disponibilidade. Isso foi possível graças à instalação da lâmpada em um Arduino Uno dotado de um dispositivo transmissor de radiofrequência nRF24L01, o qual envia informações para o gateway que implementa, de maneira transparente, a integração proposta com as modificações no knotd e o desenvolvimento do IoT Agent, permitindo assim disponibilizar as informações acerca do estado da lâmpada no Orion. Por fim, a entidade da lâmpada modelada no Orion contém atributos que, ao serem modificados, geram uma notificação que avisa à KNoT sobre a mudança, acompanhada desses dados. Após a KNoT receber os dados, ela imediatamente encaminha as informações para os dispositivos através de radiofrequência ou qualquer outro meio sendo utilizado para a comunicação. 


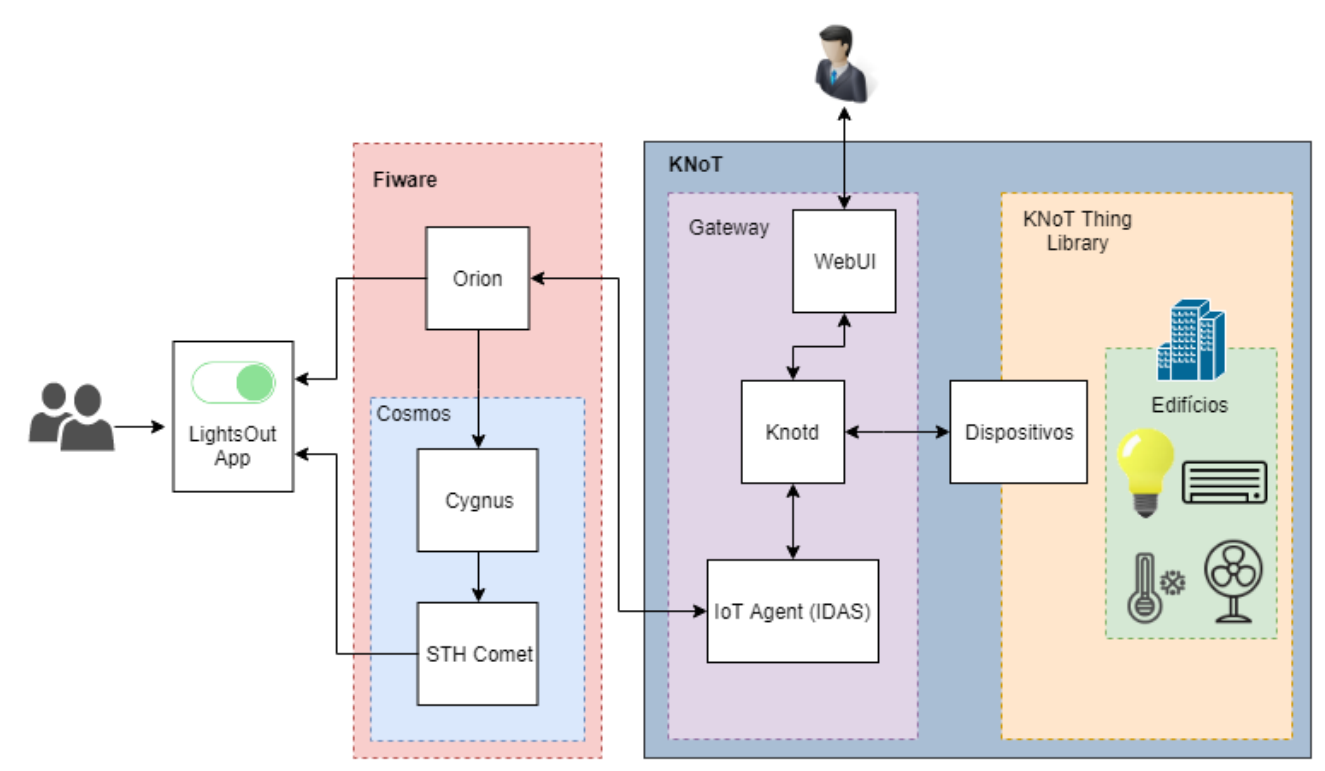

Figura 4. Arquitetura da aplicação que utiliza a integração entre KNoT e FIWARE

Partindo de uma visão global do ambiente FIWARE, foi incorporada à aplicação o uso de uma ferramenta do GE Cosmos, chamada STH Comet, cuja função é fornecer dados históricos e séries temporais agregadas por meio de uma API. O STH Comet trabalha em conjunto com o Cygnus, também componente do Cosmos, encarregado de persistir dados de contexto do Orion e criar uma visão histórica desses dados que podem posteriormente ser lidos. Para tirar proveito dessa funcionalidade, a LightsOut consulta a API do STH Comet e exibe gráficos acerca do histórico da iluminação da sala.

Apesar de ser uma aplicação com funcionalidade simples quando observada de maneira isolada, a LightsOut exemplifica o uso da solução que integra as duas plataformas abordadas. O uso adicional dos GEs do FIWARE - neste caso o Cosmos ilustra o potencial de incremento que esse ecossistema oferece para os desenvolvedores criarem novas aplicações de IoT.

\section{Trabalhos Relacionados}

O desenvolvimento de plataformas de middleware para IoT tem atraído atenção da indústria e da academia pela capacidade que esse tipo de solução tem para endereçar problemas de heterogeneidade a nível de implantação física e dos tipos de dados utilizados para comunicação entre componentes. Esses desafios vêm das especificidades dos dispositivos e se tornam barreiras para ampla adoção do paradigma. Portanto, para promover a interoperabilidade e abstração dessas particularidades, é necessário que as plataformas atendam a diversos requisitos considerados importantes nesse cenário.

Pires et al. (2015) analisaram onze plataformas para IoT e reportaram como cada uma atende aos seguintes requisitos: (i) interoperabilidade entre dispositivos, serviços e aplicações, (ii) gerenciamento e descoberta dinâmica de dispositivos, (iii) coleta, gerenciamento e processamento de informações de contexto, (iv) escalabilidade para gerenciar a crescente quantidade de dispositivos, (v) gerenciamento de grandes volumes de dados gerados pelos dispositivos físicos e transmitidos através da rede, (vi) segurança, integridade e privacidade de dados e (vii) adaptação dinâmica para garantir a disponibilidade e qualidade de aplicações em tempo de execução. Os autores 
concluíram que nenhuma delas oferece uma solução completa de maneira que atenda a todos os requisitos. Em um levantamento posterior, feito por Batista et al. (2016), outras quatro plataformas foram analisadas em complemento a esse estudo, a saber, $\mathrm{Kaa}^{4}$, SOFIA $^{5}$, FIWARE e CityHub [Lea et al. 2014]. Como resultado, constatou-se que apenas a plataforma FIWARE foi capaz de satisfazer os requisitos.

Dado que o foco do presente trabalho é analisar a facilidade da implantação física de dispositivos nas plataformas em conjunto com a interoperabilidade dos seus dados, uma solução satisfatória não foi encontrada nas outras iniciativas. Nos dois estudos mencionados, observou-se que as plataformas atendem apenas a subconjuntos dos requisitos e os abordam de formas diversas. Sobre a questão da heterogeneidade de dispositivos, a maioria das plataformas oferece suporte a protocolos comumente utilizados em IoT, como CoAP e MQTT, exigindo que os dispositivos possuam conexão direta à Internet ou o uso de um gateway para os que não têm essa capacidade. Em casos como esse, apesar da maior flexibilidade, é necessário adaptar os equipamentos para se comunicarem com o gateway e isso não é uma tarefa trivial. Plataformas como EcoDiF [Pires et al. 2014], WoT Enabler [Gao et al. 2011] e a própria FIWARE se valem desse mecanismo, porém há um esforço de integrar cada dispositivo com suas particularidades. A KNoT, por sua vez, poupa os desenvolvedores de aplicações desse trabalho adicional de elaborar a comunicação entre os dispositivos e o gateway, de modo que, ao usarem a infraestrutura fornecida pela KNoT, eles não terão que produzir drivers individuais para cada tipo de dispositivo a ser implantado. Como foi mostrado com a solução proposta neste trabalho, a integração entre as plataformas KNoT e FIWARE destaca-se pela simplicidade que a implantação de novos dispositivos pode ocorrer, favorecendo o acesso deles ao ambiente diverso que os GEs oferecem, os quais desempenham papel significativo no cumprimento dos requisitos citados acima.

\section{Conclusão}

Este artigo apresentou a integração das plataformas FIWARE e KNoT para permitir o desenvolvimento de aplicações de IoT. Esta proposta passa a oferecer a possibilidade de cooperação entre as duas plataformas, visto que agora é possível conectar dispositivos normalmente sem conexão à Internet (tais como os dotados de transmissão por radiofrequência) para serem virtualizados na FIWARE. O benefício desta contribuição se dá pelos novos tipos de dispositivos que os desenvolvedores de aplicações sobre a FIWARE podem utilizar em suas soluções, com mínimo esforço para sua implantação, sem falar que as informações providas por esses dispositivos passam a estar disponíveis para aplicações diversas.

Para fins de validação inicial da integração, foi desenvolvida uma aplicação para controlar a iluminação de uma sala e exibir estatísticas históricas sobre o uso de lâmpadas. Futuramente, serão desenvolvidas aplicações mais complexas para emprego da proposta de integração em maior escala. Ademais, a questão do desenvolvimento em conjunto com GEs de segurança da FIWARE será explorada, considerando que é um aspecto fundamental na disponibilização de aplicações para um grande número de usuários.

\footnotetext{
${ }^{4}$ https://www.kaaproject.org/

${ }^{5} \mathrm{http}: / /$ sofia2.com/home_en.html
} 


\section{Referências}

Atzori, L., Iera, A., Morabito, G. (2010) "The Internet of Things: A survey”, Computer networks 54(15), pp. 2787-2805.

Batista, T. et al. (2016) Requisitos e plataformas de middleware para cidades inteligentes. Relatório técnico - Instituto Metrópole Digital, Universidade Federal do Rio Grande do Norte, Natal, Brasil. Disponível em https://goo.gl/UMf1s4.

Cavalcante, E., Pereira, J., Alves, M. P., Maia, P., Moura, R., Batista, T., Delicato, F. C., Pires, P. F. (2016) "On the interplay of Internet of Things and Cloud Computing: A systematic mapping study”, Computer Communications 89-90, pp. 17-33.

Gao, L., Zhang, C., Sun, L. (2011) "RESTful Web of Things API in sharing sensor data", Proceedings of the 2011 International Conference on Internet Technology and Applications. USA: IEEE, pp. 1-4.

Lea, R., Blackstock, M., (2014) "City hub: A cloud-based IoT platform for smart cities". Proceedings of the IEEE 6th International Conference on Cloud Computing Technology and Science. USA: IEEE, pp. 799-804.

Mineraud, J., Mazhelis, O., Su, X., Tarkoma, S. (2016) “A gap analysis of Internet-ofThings platforms”, Computer Communications 89-90, pp. 5-16.

Miorandi, D., Sicari, S., De Pellegrini, F., Chlamtac, I. (2012) "Internet of Things: Vision, applications and research challenges", Ad Hoc Networks 10(7), pp. 14971516.

Ngu, A. H., Gutierrez, M., Metsis, V., Nepal, S., Sheng, Q. Z. (2017) "IoT middleware: A survey on issues and enabling technologies", IEEE Internet of Things Journal 4(1), pp. 1-20.

Pires, P. F., Cavalcante, E., Barros, T., Delicato, F. C., Batista, T., Costa, B. (2014) “A platform for integrating physical devices in the Internet of Things", Proceedings of the 12th IEEE International Conference on Embedded and Ubiquitous Computing. USA: IEEE, pp. 234-241.

Pires, P. F., Delicato, F., Batista, T., Barros, T., Cavalcante, E., Pitanga, M. (2015) Plataformas para a Internet das Coisas. In: Martinello, M., Robeiro, M. R. N., Rocha, A. A. A. (org.) XXXIII Simpósio Brasileiro de Redes de Computadores e Sistemas Distribuídos - Minicursos. Porto Alegre, Brasil: SBC, pp. 110-169. 